\title{
Obesity and postgastrectomy outcomes: large risks, fat chances, or no big deal?
}

\author{
Roderich E. Schwarz
}

Published online: 21 February 2012

(c) The International Gastric Cancer Association and The Japanese Gastric Cancer Association 2012

Many Western and Asian societies face an increasing prevalence of obesity and related metabolic disorders [1]. Aside from the related issues that affect health care systems in general, surgeons specifically are faced with an increasing challenge to maintain high-quality care with good outcomes in an ever-increasing number of obese patients. Overweight patients are at greater risk for comorbidity and disability, particularly at body mass indices (BMI) of greater than $35 \mathrm{~kg} / \mathrm{m}^{2}$ [2]. It is thus clear that operative care of obese patients requires greater health care resources, and frequently leads to increased length of hospital stay [3]. Associations between obesity and postoperative morbidity are less clear-cut, although many reports suggest that this is indeed so [4]. Some recent reports suggest a link between obesity and surgical site infection risk [5], while others indicate increased risks for minor complications after major cancer operations, but not major complications or death [6].

In this issue of Gastric Cancer, Sugisawa et al. [7] present a series of 206 patients undergoing curative-intent gastrectomy, in whom different fat areas as measurable components of obesity were examined for their association with postgastrectomy outcomes. The postoperative outcomes appear excellent, with low postoperative morbidity and mortality, and an acceptable (i.e., short) hospital stay for any health care system. The authors were able to link the visceral fat area (VFA), but not other measurements

This editorial refers to the article doi:10.1007/s10120-011-0099-0.

\section{R. E. Schwarz ( $\square)$}

Division of Surgical Oncology, Department of Surgery,

UT Southwestern Medical Center,

5323 Harry Hines Blvd, Dallas, TX 75390-8548, USA

e-mail: Roderich.Schwarz@utsouthwestern.edu (total or subcutaneous fat areas, BMI), to pancreas-related infections that occurred in $8.7 \%$ of patients and anastomotic leaks seen in $4.9 \%$ of patients. The authors conclude that excessive visceral fat as represented by VFA is an independent risk factor for these untoward events, and that greater diligence is required in these patients. Can we be certain about the findings and the interpretation provided, and which aspects or conclusions are relevant to current or future care of obese patients with gastric cancer?

To a "Western" reader, an anastomotic leak rate of 4.9\% appears low, but a pancreas-related infection rate of $8.7 \%$ rather high, especially since Clavien grade 1 pancreatic "fistulae" are excluded. One would agree with the authors to primarily focus on clinically relevant grades (i.e., excluding grade 1), and could suspect that the radicality of the regional dissection with splenectomy may be a causative factor. We have learned that, in contrast to Asian patients, splenectomy in Western patients can be linked to increased postoperative morbidity, especially infectious complications [8]. In fact, splenectomy (and pancreatectomy) need to be considered as causative factors for the greater morbidity and mortality observed in both Western randomized controlled trials of D2 lymphadenectomy for gastric cancer [9]. Data from Sugisawa and colleagues now suggest that this is indeed the case for their patients, but that larger VFA and increased blood loss are additional identifiable links. Is an increased VFA in a patient population with a mean BMI of 23 (rather low for many Western institutions) really reflective of increased surgical risks, and does a gradual increase of the VFA number really correlate with a significantly increased complication rate? The statistical methods the authors applied would indicate so, as VFA and other fat measurements were evaluated as continuous variables. This means that, unfortunately, there seems to be no critical cutoff value 
above which the risks increase. Importantly, the authors' results generally corroborate findings from other series in comparable patient cohorts, in which VFA was found to be a superior determinant to other fat measurements of blood loss and pancreatic fistula [10], in addition to infection, death, and longer hospital stay [11].

What then is the mechanism for such an effect? Is the VFA perhaps just a surrogate for an increased operative challenge (i.e., worse exposure, anatomic delineation, or alike), are there specific physiologic implications of increased visceral fat (but not other fat compartments) regarding inferior healing, are there oncologic factors leading to reduced recovery in the setting of increased visceral fat, or are we witnessing a combination of any or all of these factors? Every surgeon has experienced the increased technical challenges that accompany obesity. In fact, body shape as measured via BMI and celiac artery distance to the anterior skin has been linked with pancreatic fistula formation after splenectomy during gastrectomy, providing some evidence for this suspected mechanism [12]. Ueda and colleagues [13] have reported on an association between VFA and operative time as well as blood loss, but not complications, indicating greater technical difficulties in this setting. High VFA and BMI can predict technical difficulties during laparoscopic gastrectomies too, leading to an increased conversion rate and more complications [14]. On the other hand, other series of laparoscopyassisted gastrectomies have failed to show a correlation between elevated VFA and technical difficulties, supporting the notion that the VFA-complication link may be related, at least in part, to other, nontechnical factors $[15,16]$. We know quite well that the obesity phenotype is genetically regulated [17], that visceral fat is associated with diabetogenic and atherothrombotic profiles of liver lipotoxicity [18], and that inflammatory gene profiles can be detected in visceral but not subcutaneous fat of more obese individuals [19]. The suspicion that visceral fat exerts different impacts regarding inflammatory response and wound-healing regulation than other fat compartments-perhaps suppressing healing pathways in some patients with increased VFA-is sensible. Finally, links between obesity, insulin and the insulin-like growth factor axis, and cancer are well established, leaving open the possibility that cancer-related biologic effects are separate in the setting of obesity, with different implications for wound healing and recovery [20].

What should we conclude from this digression and from the data provided by Sugisawa et al.? Firstly, the findings are hypothesis-generating, and should lead us to investigate the predictive role of VFA or BMI in postoperative outcome further, especially in Western centers, where general obesity is more prevalent. Secondly, we need to be able to address the technical aspects related to greater VFA. Is it sensible to conclude that greater diligence is recommended in patients with higher VFA? One would suspect that in their series, the authors applied the greatest diligence feasible already to any operation performed, irrespective of VFA or BMI. If anything, the indications for splenectomy or splenopancreatectomy ought to be reviewed, although this was utilized selectively in the present report. We, for instance, have adopted a spleen-sparing approach to gastrectomy and extended lymphadenectomy whenever feasible, with a splenic hilar dissection for patients with proximal greater curvature and midbody primaries [21]. Even in the setting where a left-sided pancreatectomy is required, spleen preservation with splenic vascular resection and a sutured fish-mouth closure of the pancreas stump have led to a very low frequency of pancreas-specific postoperative complications [22]. Some centers would advocate to further extend the laparoscopic approach to gastrectomy due to the possibility that obesity-related technical aspects are less detrimental in this setting compared to an open approach [15]. Finally, it appears to be a good time for surgeons to get more involved in the analysis of genetic regulators of physiologic mechanisms for operative injury response and postoperative recovery, since we are increasingly gaining insight that surgical complications are genetically determined. Let us look at just two examples out of many: septic shock risk based on angiotensin II type 1 receptor polymorphisms [23], and multisystem organ dysfunction after thoracoabdominal aortic aneurysm repair [24]. Is the visceral fat compartment specifically holding gene-regulatory clues that allow us to adjust operative interventions or care in order to achieve better outcomes? If so, there seems to be promising potential for individualizing surgical therapy in the future, but only surgeons who respect the visceral fat implications and conduct tissue-based mechanistic research will hold the key to solving this concept. The risks for such a strategy do not seem too large, the chances are, let's say, "supple," and successful progress could turn into a really huge deal indeed.

\section{References}

1. Yamori Y. Worldwide epidemic of obesity: hope for Japanese diets. Clin Exp Pharmacol Physiol. 2004;31(Suppl 2):S2-4.

2. Wee CC, Huskey KW, Ngo LH, et al. Obesity, race, and risk for death or functional decline among medicare beneficiaries: a cohort study. Ann Intern Med. 2011;154(10):645-55.

3. Gedaly R, McHugh PP, Johnston TD, Jeon H, Ranjan D, Davenport DL. Obesity, diabetes, and smoking are important determinants of resource utilization in liver resection: a multicenter analysis of 1029 patients. Ann Surg. 2009;249(3):414-9.

4. Doyle SL, Lysaght J, Reynolds JV. Obesity and post-operative complications in patients undergoing non-bariatric surgery. Obes Rev. 2010;11(12):875-86.

5. Waisbren E, Rosen H, Bader AM, Lipsitz SR, Rogers SO Jr, Eriksson E. Percent body fat and prediction of surgical site infection. J Am Coll Surg. 2010;210(4):381-9. 
6. Mullen JT, Davenport DL, Hutter MM, et al. Impact of body mass index on perioperative outcomes in patients undergoing major intra-abdominal cancer surgery. Ann Surg Oncol. 2008; 15(8):2164-72.

7. Sugisawa N, Tokunaga M, Tanizawa Y, Bando E, Kawamura T, Terashima M. Intra-abdominal infectious complications following gastrectomy in patients with excessive visceral fat. Gastric Cancer. 2011.

8. Brady MS, Rogatko A, Dent LL, Shiu MH. Effect of splenectomy on morbidity and survival following curative gastrectomy for carcinoma. Arch Surg. 1991;126(3):359-64.

9. Kodera Y, Schwarz RE, Nakao A. Extended lymph node dissection in gastric carcinoma: where do we stand after the Dutch and British randomized trials? J Am Coll Surg. 2002;195(6): 855-64.

10. Tanaka K, Miyashiro I, Yano M, et al. Accumulation of excess visceral fat is a risk factor for pancreatic fistula formation after total gastrectomy. Ann Surg Oncol. 2009;16(6):1520-5.

11. Tokunaga M, Hiki N, Fukunaga T, Ogura T, Miyata S, Yamaguchi $\mathrm{T}$. Effect of individual fat areas on early surgical outcomes after open gastrectomy for gastric cancer. Br J Surg. 2009;96(5): 496-500.

12. Yamamoto N, Oshima T, Sato T, et al. Upper abdominal body shape is the risk factor for postoperative pancreatic fistula after splenectomy for advanced gastric cancer: a retrospective study. World J Surg Oncol. 2008;6:109.

13. Ueda J, Ichimiya H, Okido M, Kato M. The impact of visceral fat accumulation on laparoscopy-assisted distal gastrectomy for early gastric cancer. J Laparoendosc Adv Surg Tech A. 2009;19(2): $157-62$

14. Kunisaki C, Makino H, Takagawa R, et al. Predictive factors for surgical complications of laparoscopy-assisted distal gastrectomy for gastric cancer. Surg Endosc. 2009;23(9):2085-93.
15. Makino H, Kunisaki C, Izumisawa Y, et al. Effect of obesity on laparoscopy-assisted distal gastrectomy compared with open distal gastrectomy for gastric cancer. J Surg Oncol. 2010;102(2): $141-7$.

16. Yoshikawa K, Shimada M, Kurita N, et al. Visceral fat area is superior to body mass index as a predictive factor for risk with laparoscopy-assisted gastrectomy for gastric cancer. Surg Endosc. 2011;25(12):3825-30.

17. Kring SI, Larsen LH, Holst C, et al. Genotype-phenotype associations in obesity dependent on definition of the obesity phenotype. Obes Facts. 2008;1(3):138-45.

18. Rocha PM, Barata JT, Minderico CS, Silva AM, Teixeira PJ, Sardinha LB. Visceral abdominal and subfascial femoral adipose tissue have opposite associations with liver fat in overweight and obese premenopausal Caucasian women. J Lipids. 2011;2011: 154672 .

19. Tam CS, Heilbronn LK, Henegar C, et al. An early inflammatory gene profile in visceral adipose tissue in children. Int $\mathrm{J}$ Pediatr Obes. 2011;6(2-2):e360-3.

20. Donohoe CL, Doyle SL, Reynolds JV. Visceral adiposity, insulin resistance and cancer risk. Diabetol Metab Syndr. 2011;3:12.

21. Schwarz RE. Spleen-preserving splenic hilar lymphadenectomy at the time of gastrectomy for cancer: technical feasibility and early results. J Surg Oncol. 2002;79(1):73-6.

22. Mohebati A, Schwarz RE. Extended left-sided pancreatectomy with spleen preservation. J Surg Oncol. 2008;97(2):150-5.

23. Nakada TA, Russell JA, Boyd JH, et al. Association of angiotensin II type 1 receptor-associated protein gene polymorphism with increased mortality in septic shock. Crit Care Med. 2011;39(7):1641-8.

24. Feezor RJ, Baker HV, Xiao W, et al. Genomic and proteomic determinants of outcome in patients undergoing thoracoabdominal aortic aneurysm repair. J Immunol. 2004;172(11):7103-9. 